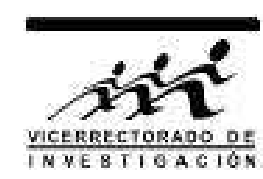

\title{
Diseño aerodinámico de un túnel de viento de bajas velocidades
}

\author{
C. A. Quispe Gonzáles*, W. J. Urcuhuaranga Esteban y J. E. Chiroque Baldera \\ E. A. P. de Ingeniería Mecánica de Fluidos, Facultad de Ciencias Físicas, \\ Universidad Nacional Mayor de San Marcos, Lima, Perú
}

Recibido 30 abril 2014 - Aceptado 18 junio 2014

\begin{abstract}
El presente trabajo tiene como objetivo desarrollar el diseño aerodinámico y la determinación de las características geométricas de un túnel de viento de baja velocidad. Fue elegido un túnel de viento abierto de tipo Eiffel, de acuerdo a las condiciones requeridas en la cámara de prueba, tales como el área de la sección de ensayo y la velocidad máxima del aire en esta zona; se propone el esquema constructivo con los elementos que conforman el túnel y la geometría de los mismos. Son efectuados los cálculos aerodinámicos para determinar la velocidad, el área de paso y las pérdidas aerodinámicas en las secciones características de la instalación. Se determina la potencia necesaria del ventilador y su selección de acuerdo a las condiciones del mercado nacional.
\end{abstract}

Palabras claves: Túnel de viento, análisis aerodinámico, admisión del túnel, contracción del túnel, sección de prueba, difusor, ventilador del túnel.

\section{Aerodynamical design of a low velocities wind tunnel}

This paper aims to develop the aerodynamical design and the geometric detrmination of a low veñocities wind tunnel. Was elected an Eiffel type open wind tunnel, according to the required conditions in the test chamber, such as the area and maximum air velocity in the test section; we propose the constructive layout with the tunnel elements and the geometry of them. We performe calculations to determine the flow veloctiy, the cross section and the aerodynamical losses in the characteristic sections of the instalation. We determine the required fan power and it's selection according to the local market conditions.

Keywords: Wind tunnel, aerodynamic analysis, tunnel admission, tunnel contraction, test section, difusor, tunnel fan.

En ingeniería, un túnel de viento o túnel aerodinámico es una instalación de investigación en el que se obtienen flujos de aire rectilíneo y uniforme a una velocidad determinada en la cámara de ensayos, se desarrolla para asistir a los estudios de los efectos del movimiento del aire alrededor de los objetos sólidos. El primer túnel de viento fue construido por Wenham en 1871 y posteriormente otros investigadores como Reynolds, Tsiolkovsky, Lilienthal, Langley, Prandtl, von Karman, entre otros, utilizaron estos túneles en sus trabajos experimentales introduciendo mejoras en su diseño [1].

Un túnel de viento llega a ser una herramienta útil en la investigación de la mecánica de los fluidos teniendo como principales ventajas la reducción del objeto de estudio a un modelo a escala, optimización en el diseño y funcionamiento del modelo, repetitividad de los ensayos, así como el tiempo y la economía, que generan ventajas frente a las pruebas que se realizan en el campo. Sin em- bargo, el túnel presenta el inconveniente del elevado costo de instalación, especialmente para el ensayo de objetivos de tamaño real. Hoy en día, la utilidad de un túnel de viento es obvia, ya existen túneles de viento de gran tamaño, capaces de ensayar modelos a escala real y que funcionan en una amplia gama de velocidades, como los túneles supersónicos. Las innovaciones introducidas, principalmente en su construcción, planta de potencia y regulación, hacen que sea altamente competitivo en costos y prestaciones, así como adecuado para una gran variedad de aplicaciones, como por ejemplo, ensayos aeronáuticos, ingeniería civil, arquitectura, energías renovables, medio ambiente, entrenamiento deportivo e investigación y desarrollo agrícola.

No existe una información apropiada respecto al diseño y la construcción de túneles de viento, debido a la diversidad de tipos de acuerdo a su configuración -circuito abierto y cerrado-, de acuerdo al régimen de trabajo subsónico, transónico, supersónico e hipersónico-, así co-

*cquispeg@gmail.com 
mo por el diseño de la sección de prueba -sección cerrada o sección abierta-. La gran mayoría de la bibliografía es de tipo descriptivo de experiencias constructivas alrededor del mundo. Pero entre la escasa bibliografía, existen trabajos importantes que son conclusiones de muchos años de trabajo experimental y que sirven de referencia para realizar proyectos de túneles de viento, como el trabajo de Barlow et al. [3], Bell y Metha [4], Metha y Bradshaw [5] y Pope y Goin [6]. Estas informaciones complementadas con conocimientos de aerodinámica y mecánica de fluidos nos permite aventurarnos en la ejecución de proyectos de túneles de viento.

En el país, existen algunos túneles de viento, los cuales generalmente son utilizados con fines didácticos en universidades e instituciones de formación profesional. La Universidad Nacional Mayor de San Marcos (UNMSM), en la Escuela Académica Profesional de Ingeniería Mecánica de Fluidos, se puede encontrar uno de estos túneles, el cual a la fecha no ha podido ser puesto en funcionamiento. Otras universidades, como la Universidad Tecnológica del Perú, con el apoyo de la cooperación española; concluyó hace poco la construcción de un túnel de viento con fines didácticos.

La organización de cooperación técnica Internacional Intermediate Technology Development Group (ITDG) ejecutó un proyecto de electrificación rural, mediante sistemas de micro-aerogeneradores individuales de $100 \mathrm{~W}$, de fabricación nacional mejorados técnicamente, fueron testados en un laboratorio en el km. 123.5 de la Panamericana Norte, Huacho, donde existe una presencia de vientos en casi todo el año y se sitúa frente al mar. En el fueron efectuados muchos ensayos con un equipo de 100 y $500 \mathrm{~W}$, los resultados obtenidos estaban más asociados a la parte eléctrica, siendo bastante dificultoso obtener resultados referidos a la parte mecánica y aerodinámica debido a que no se podía controlar el régimen del viento. Además, los gastos asociados a la parte experimental eran onerosos, debido al elevado costo de estadía y pérdida de horas hombre, ya que había ocasiones en que no soplaba el viento.

Esta situación llevó a la necesidad de contar con un túnel de viento que permita simular condiciones de flujos de aire con bajas velocidades, que permitan simular condiciones de viento a diferentes velocidades y regímenes de trabajo, siendo uno de los motivos el desarrollo del presente trabajo. ITDG firmó un convenio con la Universidad Nacional de Ingeniería UNI para instalar y poner en operación este túnel de viento con cámara de ensayo de 1.20 $\mathrm{m}$ y que actualmente se encuentra operativo en el campus universitario de la UNI. Cabe mencionar, que el diseño, construcción e instalación del citado túnel estuvo a cargo de los autores de este paper, quienes pertenecen a la UNMSM.

\section{Fundamentos teóricos}

Un túnel de viento es un dispositivo bastante simple. La mayoría de los diseños tienen seis componentes o secciones características como son las zonas de admisión, de estabilización, de contracción, de prueba o de ensayo, el difusor y la unidad de potencia o ventilador. Estas partes se muestran en la Fig. 1 1 El diseño total, crea un flujo de aire a alta velocidad y de baja turbulencia, que es dirigido a través de la sección de prueba y permite que en los procesos de investigación se midan las interacciones, que producen el conjunto flujo de aire-modelo, como las fuerzas resultantes, los coeficientes aerodinámicos, los efectos de turbulencia, entre otros.

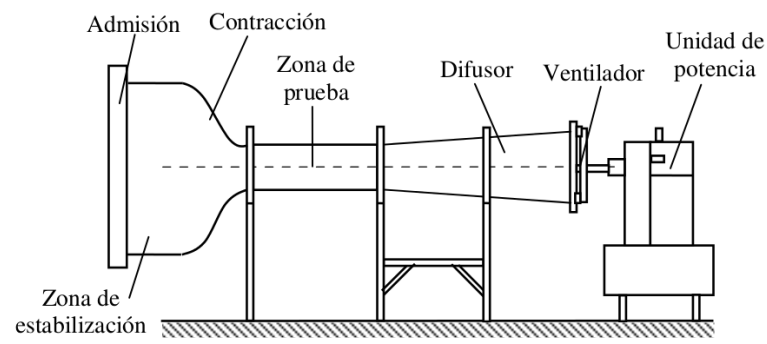

Figura 1: Partes características de un túnel de viento de tipo abierto.

Cada componente o sección característica realiza una función en el esquema, que se explica brevemente a continuación. La zona de admisión es prácticamente la entrada al conducto principal del túnel y su función es evitar la desestabilización de las corrientes de aire y la formación y propagación de las turbulencia que se deben a cambios abruptos de la velocidad del viento. La zona de estabilización es una zona de área constante en donde se encuentran el corrector del flujo y las rejillas estabilizadoras. Permite que el flujo entrante pueda pasar a la zona de contracción como un flujo uniforme y de poca turbulencia. La zona de contracción es una zona donde ocurre la disminución del área de paso del túnel y su función es acelerar el flujo y disminuir las variaciones de velocidad. En esta zona, el fluido se acelera hasta alcanzar la velocidad de diseño en la zona de prueba. La sección de trabajo o sección de prueba es la zona donde normalmente se instala el modelo de estudio y en donde la velocidad del flujo alcanza su régimen de prueba, siendo las líneas de corriente uniformes y paralelas. El difusor tiene la función principal de disminuir la velocidad de salida del flujo, gracias a un incremento del área de paso. Con esto, se aumenta paulatinamente la presión y se disminuye la velocidad del flujo, lo cual hace disminuir las pérdidas por fricción. Finalmente, el aire por si solo no va a pasar espontáneamente por el tunel de viento. Para que el aire atraviese el tunel es necesario inducir al aire a atravesar el túnel. Esto se logra con una unidad de potencia, que en la mayoría de los casos es un ventilador, que proporciona la fuerza necesaria para mover 
el aire a través del túnel de viento.

Existen varias maneras de clasificar los túneles de viento. Atendiendo al tipo de configuración, los túneles pueden ser: a) abierto, en donde un ventilador succiona el aire a través del conducto del túnel y luego, expulsa el aire de nuevo al exterior; b) cerrado, llamado también túnel de recirculación porque el aire es forzado a circular en forma indefinida por el interior del túnel. Otra forma de clasificación de los túneles es de acuerdo a su régimen de trabajo, es decir, a la velocidad del aire en la zona de prueba, aquí se clasifican como: a) subsónicos, en donde la velocidad del aire en la zona de prueba no supera la velocidad crítica con número de Mach menores de 0.7, $M<0.7$ ); b) transónicos con $0.7<M<1.2$; c) supersónicos con $1.2<M<5$; y d) hipersónicos con $M>5$. Por el diseño de la sección de prueba, los túneles de viento se clasifican como: a) con sección de ensayo cerrada, donde la sección de prueba es una continuación de la sección de salida de la zona de contracción y está limitada por paredes siendo generalmente una zona de sección constante; b) con sección de ensayo abierta, donde la sección de ensayos no está confinada por las paredes, el aire del interior del túnel se mezcla con el exterior, disminuyendo casi totalmente el problema de errores introducidos debido a la proximidad de las paredes al modelo y su interferencia con el flujo.

Además de los aspectos descritos para el diseño de un túnel de viento, cabe mencionar la influencia del modelo de prototipo que se pretende ensayar. Las paredes del túnel deben estar bastante alejadas del modelo de prototipo para tener un perfil de flujo bastante similar al real, puesto que si el cociente entre el área frontal del modelo y el área de la sección de ensayos es mayor que 0.075 -otros autores sugieren 0.1-, la obstrucción que supone el modelo al paso del aire a mayores velocidades del flujo entre el modelo y las paredes determina que los coeficientes de sustentación y de arrastre estimados se desvían de los valores reales [7]. Por ello, es deseable que el área de la sección de ensayos sea lo mayor posible, pero a mayor área, el costo de la construcción y la potencia necesaria en el ventilador aumentan considerablemente. Todo esto hace que el tamaño del modelo sea condicionado por el tamaño de la sección de ensayos disponible y por el límite del cociente de las áreas frontales del modelo y la sección de ensayos. El bloqueo del flujo ocurre durante las pruebas con modelos relativamente grandes en la sección de túneles de tamaño limitado. Este bloqueo se define como el radio de la sección frontal del modelo al área de la sección de prueba. Se necesitan radios de bloqueo menores del $10 \%$ de la sección a pesar de que muchas veces se excede este límite con creces. Para las pruebas aerodinámicas, este bloqueo no debe ser mayor que el $5 \%$. La presencia del modelo en la sección de prueba tiene como resultado que al bloquear el flujo aumenta la presión en las paredes del túnel. Por esta razón, los túneles de sección abierta se emplean a menudo. Las correcciones por bloqueo son todavía un tema de gran interés de investigación.

El movimiento del flujo compresible isoentrópico, que pasa por el túnel de viento, puede escribirse como [8],

$$
V d V+\frac{d p}{\rho}+g d z=0
$$

siendo $V$ es la velocidad del flujo, $p$ es la presión estática, $\rho$ la densidad, $z$ es la altura respecto a un plano de referencia y $g$ es la constante gravitacional. Para muchos procesos que envuelven gases, las fuerzas gravitacionales pueden ser despreciadas $g d z \approx 0$. Para el proceso isoentrópico, $p / \rho^{k}=$ const, la Ec. (1) se integra resultando

$$
\frac{V^{2}}{2}+\int \frac{d p}{\rho}=\text { const } \Rightarrow \frac{V^{2}}{2}+\frac{k}{k-1} \frac{p}{\rho}=\text { const } .
$$

Si se considera que el flujo es incompresible $\rho=$ const, la Ec. (2) se transforma en

$$
\frac{V^{2}}{2}+\frac{p}{\rho}=\text { const } .
$$

Para el caso de un flujo adiabático sin rozamiento, la velocidad local del sonido se expresa como $a^{2}=(\partial p / \partial \rho)$. Considerando un gas ideal con $p / \rho=R T$, se expresa como una función continua de dos variables de la forma $z=f(x, y)$, además de las relaciones de Maxwell; la velocidad local del sonido a estas condiciones queda definida como [9]

$$
\begin{aligned}
a^{2} & =\left(\frac{\partial p}{\partial \rho}\right)_{s}=k\left(\frac{\partial p}{\partial \rho}\right)_{T} \\
a & =\sqrt{k R T}=\sqrt{k p / \rho} .
\end{aligned}
$$

El número de Mach se define como la relación entre la velocidad del flujo y la velocidad local del sonido, $M=V / a$, relacionando la energía cinética del flujo con la energía potencial o energía molecular del gas, expresada a través de la velocidad local del sonido. Se debe indicar, que tanto $V$ como $a$, cambian de sección en sección por ser de carácter local. Los parámetros de estancamiento del flujo pueden ser expresados a través del número de Mach mediante las siguientes relaciones [8],

$$
\begin{aligned}
& \frac{T_{o}}{T}=1+\frac{k-1}{2} M^{2}, \\
& \frac{p_{o}}{p}=\left(1+\frac{k-1}{2} M^{2}\right)^{\frac{k}{k-1}}, \\
& \frac{\rho_{o}}{\rho}=\left(1+\frac{k-1}{2} M^{2}\right)^{\frac{1}{k-1}} .
\end{aligned}
$$

Los parámetros críticos del flujo, pueden ser expresados a 
través de las relaciones

$$
\begin{aligned}
\frac{T^{*}}{T_{o}} & =\frac{2}{k+1} M^{2}, \\
\frac{p^{*}}{p_{o}} & =\left(\frac{2}{k+1}\right)^{\frac{k}{k-1}}, \\
\frac{\rho^{*}}{\rho_{o}} & =\left(\frac{2}{k+1}\right)^{\frac{1}{k-1}} .
\end{aligned}
$$

Otro criterio usado y similar al número $M$, es el coeficiente de velocidad $\lambda \circ$ Mach crítico $M^{*}$, que relaciona la velocidad del flujo con su velocidad crítica $\lambda=M^{*}=$ $V / a^{*}$. Como en el flujo adiabático $T_{o}=$ const, físicamente el coeficiente de velocidad $\lambda$ relaciona la energía cinética del flujo con su energía total, expresada a través de la temperatura de estancamiento. La relación que existe entre $\lambda$ y $M$ se determina mediante

$$
\begin{gathered}
\lambda=\frac{V}{a^{*}}=\frac{V}{a} \frac{a}{a_{o}} \frac{a_{o}}{a^{*}}=M\left(1+\frac{k-1}{2} M^{2}\right)^{1 / 2} \times \\
\left(\frac{2}{k+1}\right)=\left[\left(\frac{k+1}{2} M^{2}\right)\left(1+\frac{k-1}{2} M^{2}\right)\right]^{1 / 2}
\end{gathered}
$$

de esta expresión, Ec. (7), se observa que $M=$ $0 \Rightarrow \lambda=0$, si $M=1 \Rightarrow \lambda=1$ y si $M=$ $\infty \Rightarrow \lambda=\sqrt{(k+1) /(k-1)}$, por lo que $0 \leq \lambda \leq$ $[(k+a) / k-1]^{1 / 2}$. La relación inversa de $M$ y $\lambda$ se determina mediante

$$
\begin{aligned}
& M=\frac{V}{a}=\frac{V}{a *} \frac{a *}{a_{o}} \frac{a_{o}}{a}=\lambda\left(\frac{2}{k+1}\right)^{1 / 2} \times \\
& \left(1-\frac{k-1}{k+1} \lambda^{2}\right)^{-1 / 2}=\left[\frac{\left(\frac{1}{k+1} \lambda^{2}\right)}{\left(1-\frac{k-1}{k+1} \lambda^{2}\right)}\right]^{1 / 2} .
\end{aligned}
$$

Los parámetros de estancamiento del flujo en función del coeficiente de velocidad, se determina transformando la Ec. (2) de la forma [8]

$$
\begin{aligned}
& \frac{T}{T_{o}}=1-\frac{k-1}{k+1} \lambda^{2} \\
& \frac{p}{p_{o}}=\left(1-\frac{(k-1)}{(k+1)} \lambda^{2}\right)^{\frac{k}{k-1}} \\
& \frac{\rho}{\rho_{o}}=\left(1-\frac{(k-1)}{k+1} \lambda^{2}\right)^{\frac{1}{k-1}}
\end{aligned}
$$

además, el gasto másico dado por $\dot{m}=\rho A V$, se expresa en función del coeficiente de velocidad $\lambda$ como [10],

$$
\begin{aligned}
\dot{m} & =\frac{p_{o} A}{\sqrt{R T_{o}}} \sqrt{k \frac{2}{k+1}} \lambda\left(1-\frac{k-1}{k+1} \lambda^{2}\right)^{\frac{1}{k-1}} \\
& =\text { const } ;
\end{aligned}
$$

y el gasto másico específico por unidad de área de un flujo a través de un conducto cualquiera, se expresa como $\bar{m}=\dot{m} / A=\rho V$, mientras que el gasto específico reducido $q$ se obtiene al expresar un gasto másico específico respecto al máximo valor del gasto específico, es decir,

$$
\begin{aligned}
& q=\frac{\bar{m}}{\bar{m}_{\mathrm{MAX}}}=\frac{\rho V}{\rho^{*} V^{*}}=\frac{\rho_{o}}{\rho^{*}} \frac{\rho}{\rho_{o}} \frac{V}{a^{*}} \\
&=\left(\frac{k+1}{2}\right)^{\frac{1}{k-1}}\left(1-\frac{k-1}{k+1} \lambda^{2}\right)^{\frac{1}{k-1}} \lambda .
\end{aligned}
$$

Esta función $q$ depende del coeficiente de velocidad $\lambda$ y del índice isoentrópico $k$, tiende a cero para dos valores, para $\lambda=0$ y $\lambda=\lambda_{\mathrm{MAX}}=\sqrt{(k+1) /(k-1)}$; por lo tanto tiene un valor extremo. Cuando $d q / d \lambda=0 \Rightarrow \lambda=1$ Esto indica que la función $q$ tiene un valor máximo para $\lambda=1$, siendo una función bivalente, esto es, $q$ tiene dos valores de $\lambda$, uno correspondiente al régimen subsónico y el otro correspondiente al régimen supersónico, tal como se muestra en la Fig. (2). La elección adecuada del valor de $\lambda$ se realiza en función de las condiciones del problema planteado.

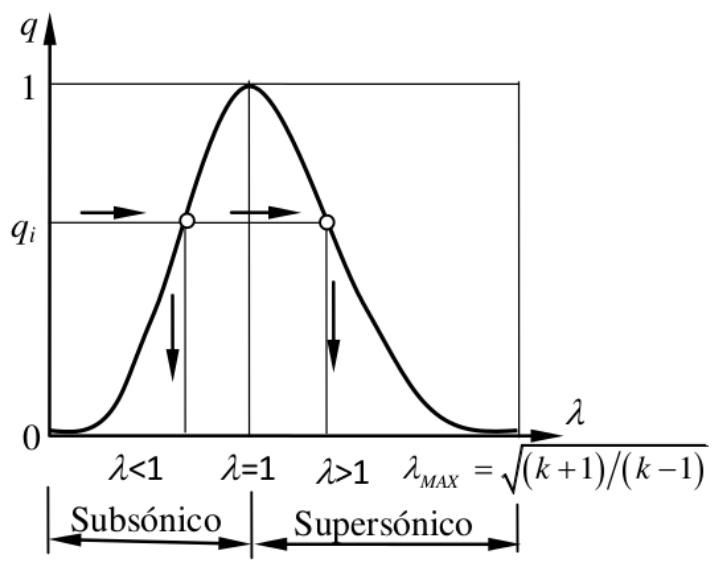

Figura 2: Dependencia del coeficiente de gasto específico $q$ reducido en función de $\lambda$.

La función $q$ permite enlazar la geometría del conducto con los parámetros del flujo. Como el gasto másico es constante en cualquier sección del túnel. Al relacionar una sección con área $A_{i}$ y su sección crítica $A^{*}$, se obtiene:

$$
\bar{m}=\rho_{i} A_{i} V_{i}=\rho^{*} A^{*} V^{*} \Rightarrow q=\frac{\rho_{i} V_{i}}{\rho^{*} V^{*}}=\frac{A^{*}}{A_{i}}
$$

así, la función $q$ además de ser una función gasodinámica es también una función geométrica, lo que permite resolver problemas en conductos de forma arbitraria.

El gasto másico $\bar{m}$ es expresado particularmente como una función del gasto másico reducido $q$. Esto es evidente 
si se hace algunas transformaciones a la definición

$$
\begin{aligned}
\bar{m}=\rho V A=\frac{\rho V A}{\rho^{*} V^{*} A^{*}} \bar{m}^{*}=q \frac{A}{A^{*}} \bar{m}^{*} \\
=q \frac{p_{o} A}{\sqrt{E T_{o}}} \sqrt{k\left(\frac{2}{k+1}\right)^{\frac{k+1}{k-1}}}
\end{aligned}
$$

y para un sistema aislado térmicamente $T_{01}=T_{02}$, la ecuación se reduce a

$$
\frac{p_{01}}{p_{02}}=\frac{A_{2}}{A_{1}} \frac{q_{2}}{q_{1}} .
$$

La ecuación de Bernoulli para el flujo compresible es más compleja que la ecuación de Bernoulli para el flujo incompresible, ya que la densidad del gas no es constante en el transcurso del flujo. Pero también es posible calcular la presión del gas utilizando las ecuaciones del flujo incompresible, siempre que el número $M$ no sea mayor que 0.3 .

Las pérdidas en los conductos del sistema son debidas a las transformaciones irreversibles que ocurren cuando se mueve el fluido, en gran medida a la transformación de energía mecánica en calor. Son dos los tipos de pérdidas que se encuentran en un sistema de ductos, las pérdidas por fricción y las pérdidas dinámicas.

Las pérdidas por fricción son debidas a la viscosidad del fluido y se producen a lo largo de toda la longitud del conducto y se calculan por la ecuación de DarcyColebrook [12],

$$
\Delta p_{f}=\frac{f L}{D_{H}} \frac{\rho V^{2}}{2}
$$

donde $\Delta p_{f}$ representa las pérdidas por fricción en términos de presión total, $f$ es el factor de fricción; $L$ es la longitud del ducto, $D_{H}$ es el diámetro hidráulico; $\rho$ es la densidad del flujo y $V$ la velocidad promedio. Dentro de la región del flujo laminar el factor de fricción es solo una función del número de Reynolds $R e$, siendo [12] $f=64 / R e$. Blasius consiguió un factor de fricción turbulento en tuberías lisas en el intervalo $4.0 \times 10^{3}<R e<10^{5}$, proponiendo la siguiente expresión $f_{\text {lisa }} \approx 0.3164 R e^{-1 / 4}$. A su vez, Prandtl dedujo el factor de fricción del régimen turbulento para tubería lisa, $1 / \sqrt{f}=2.0 \log [\operatorname{Re} \sqrt{f}]-0.8$. Von Karman desarrolló una expresión para un flujo completamente turbulento en tuberías muy rugosas de la forma $1 / \sqrt{f}=-2.0 \log [(\varepsilon / D) / 3.7]$. Para un flujo completamente turbulento, el factor de fricción depende del número de $R e$, la rugosidad de la superficie del conducto y de las protuberancias internas tales como juntas, articulaciones. En la zona de transición rugosa el coeficiente de fricción es calculado por la ecuación de Colebrook,

$$
\frac{1}{\sqrt{f}}=-2 \log \left[\frac{\varepsilon}{3.7 D_{H}}+\frac{2.51}{R e \sqrt{f}}\right],
$$

siendo $\varepsilon$ el factor de rugosidad absoluta del material, en $\mathrm{mm}$ y $R e$ el número de Reynolds. El número de Reynolds es dado por $R e=D_{H} V / v$, donde $v$ es la viscosidad cinemática.

Las pérdidas dinámicas aparecen como resultado de las perturbaciones del flujo causados por equipos montados en los conductos y por los accesorios que cambian la dirección de la trayectoria del flujo de aire y de área. Estos accesorios incluyen entradas y salidas, codos, transiciones, cruces, etc. Una amplia investigación de estos fenómenos con el fin de determinar los coeficientes de pérdidas han llevado a obtener resultados en tres formas, tablas, curvas y ecuaciones [13].

Coeficiente de pérdida local. El coeficiente adimensional $k_{p}$ es usado para definir la pérdida de presión en un conducto porque este coeficiente tiene el mismo valor en corrientes dinámicas similares, es decir, secuencias con tramos geométricamente similares, iguales números de $\operatorname{Re} e$ iguales valores de otros criterios necesarios para la similitud dinámica. Está basado sobre la relación de la pérdida de presión total respecto a la presión de velocidad en la sección transversal a la que se hace referencia, es decir,

$$
K_{p}=\frac{\Delta p_{j}}{\rho V^{2} / 2}=\frac{\Delta P_{j}}{p_{v}},
$$

siendo $\Delta p_{j}$ la pérdida total de presión, $\rho$ la densidad del fluido, $V$ la velocidad del fluido y $p_{v}$ la presión dinámica. Para todos los accesorios, excepto los cruces, se puede calcular la pérdida total de presión $\Delta p_{j}$ en una sección mediante $\Delta p_{j}=K_{0} p_{v, 0}$, siendo que el subíndice 0 está referido a la sección transversal en la que se hace referencia a la presión dinámica $p_{v, 0}$. También se debe indicar que el flujo compresible puede ser tratado como flujo incompresible siempre y cuando la velocidad del flujo no supere a su correspondiente número $M=0.3[8]$.

\section{Consideraciones geométricas del diseño}

Debemos considerar las condiciones iniciales del flujo a la entrada del túnel, pues el fluido a utilizar es el aire atmosférico y dado que la altitud sobre el nivel del mar del lugar de instalación es relativamente pequeña como para ser tomada en cuenta, las propiedades del aire atmosférico serán tomadas a condiciones estándar a nivel del mar, teniendo los siguientes datos de entrada [11],

\begin{tabular}{ll}
\hline Presión atmosférica & $p_{0}=101.325 \mathrm{kPa}$ \\
Temperatura atmosférica & $T_{0}=20^{\circ} \mathrm{C}=293 \mathrm{~K}$ \\
Densidad & $\rho=1.225 \mathrm{~kg} / \mathrm{m}^{3}$ \\
Constante del aire & $R=287 \mathrm{~J} / \mathrm{kg}-\mathrm{K}$ \\
Índice adiabático del aire & $c_{p}=1.005 \mathrm{~J} / \mathrm{kg}-\mathrm{K}$ \\
Calor específico & $k=1.4$ \\
Viscosidad cinemática & $v=1.46 \times 10^{-5} \mathrm{~m}^{2} / \mathrm{s}$ \\
\hline
\end{tabular}

Las condiciones iniciales en la zona de prueba o cámara de prueba están dadas por 


\begin{tabular}{|c|c|}
\hline Velocidad de diseño del flujo & $14 \mathrm{~m} / \mathrm{s}$ \\
\hline $\begin{array}{l}\text { Diámetro del área frontal del } \\
\text { modelo de ensayo }\end{array}$ & $1.0 \mathrm{~m}$ \\
\hline Diámetro a la salida del colector & $1.4 \mathrm{~m}$ \\
\hline
\end{tabular}

\section{Determinación de las dimensiones de la cámara de prueba}

Debido al tamaño del modelo, la cámara de prueba debe de ser de tal tamaño, que las paredes no influyan sobre el modelo durante los ensayos y no introduzcan errores en las mediciones. El bloqueo del flujo ocurre durante las pruebas con modelos relativamente grandes en la sección de túneles de tamaño limitado. Este bloqueo se define como el radio de la sección frontal del modelo al área de la sección de prueba. Se necesitan radios de bloqueo menores del $10 \%$ de la sección a pesar de que muchas veces esto se excede con creces. Por esta razón, los túneles de sección abierta se emplean a menudo. El coeficiente de bloqueo debe encontrarse entre 0.075 y 0.10 [7]. Así tenemos que

$$
\begin{aligned}
\frac{\text { área frontal del modelo }}{\text { área de la zona de prueba }} & =\left(\frac{d_{M}}{d_{Z P}}\right) \\
& \in[0.075-0.10],
\end{aligned}
$$

siendo $d_{M}$ el diámetro frontal del modelo, que es igual a $1.00 \mathrm{~m} \mathrm{y} d_{Z P}$ el diámetro de la zona de prueba. Entonces: $\left(10 / d_{Z P}\right)=[0.075-0.10] \Rightarrow d_{Z P} \in[3.10-3.65]$ $\mathrm{m}$. Bajo este criterio, se optó por una sección de prueba con diámetro hidráulico $D_{H}=3.5 \mathrm{~m}$. También se optó por una sección de prueba de forma cuadrada, lo que facilita la instalación del modelo al tener un piso horizontal y para instalar los instrumentos de medición. La longitud de la cámara de prueba requiere de la definición previa del diámetro de la sección de salida del colector y que es anterior a la zona de prueba. Se optó por un diámetro a la salida del colector $d_{s, c}$ con un área cerca del $200 \%$ del modelo, para evitar que la desuniformidad del flujo pueda afectar el trabajo del modelo, este mismo diámetro debe ser mantenido a la entrada del difusor. Así tenemos

$$
\left(\frac{d_{M}}{d_{S, C}}\right)^{2}=2.0 \Rightarrow d_{S, C}=1.414 d_{M}=1.414 \mathrm{~m},
$$

finalmente, se optó como diámetro de salida del colector $d_{S, C}=1.40 \mathrm{~m}$.

La longitud de la cámara de prueba, está en función de la longitud del modelo y la distancia necesaria para eliminar el efecto de una expansión brusca. Según recomendaciones [12] el flujo al salir a una expansión brusca se expande ocupando el mayor área posible. Al ser la cámara de prueba una instalación estanca bajo similares condiciones de presión, las líneas de corriente del flujo tratarán de expandirse al salir del colector. No existe información sobre la longitud de reacomodo de las líneas de corriente, pero en casos de ductos se asume que esta expansión ocurre a una distancia no mayor que el diámetro menor que conforma la expansión brusca e igual a $d_{S . C}$. Similar situación se tiene para la contracción brusca que se originará a la entrada del difusor. Así, la longitud de la cámara de prueba $l_{C . P}=2 l_{C . P}+l_{M}=4.10 \mathrm{~m}$, optándose finalmente por $l_{C . P}=4.50 \mathrm{~m}$. Así, la cámara estanca que es la zona de prueba, tendrá una sección cuadrada de $3.50 \times 3.50 \mathrm{~m}^{2}$ y una longitud de $4.50 \mathrm{~m}$.

\section{Consideraciones para el diseño del colector}

El colector del túnel de viento propuesto está conformado por la admisión, la zona de estabilización y de contracción, a través del cual se lleva un flujo uniforme a la zona de prueba. Generalmente, el diseño de la contracción es la parte más importante del circuito del túnel de viento, aquí el flujo se acelera rápidamente, las líneas de corriente están sometidas a una gran tensión, lo que reduce las variaciones del flujo medio y genera una gran razón de contracción de las mismas. Pero, por ser un túnel de baja velocidad, estas condiciones se atenúan. La decisión de tener una entrada curva y de instalar una rejilla estabilizadora obedece a la necesidad de disminuir las pérdidas por estabilización del flujo en el colector.

La razón de contracción se encuentra entre 6 a 9 para túneles pequeños [5]. Debido a que las velocidades para el presente proyecto no superan los $14 \mathrm{~m} / \mathrm{s}$, el problema de la separación de la capa límite puede ser contrarrestada si es que se elige paredes con superficie de curvatura convexa, sin puntos de inflexión, en donde el área de la sección transversal irá disminuyendo suave y monótonamente, siendo conveniente elegir un perfil de forma parabólica. Así el diámetro efectivo de entrada al colector es

$$
\begin{aligned}
\left(\frac{d_{E . C}}{d_{S . C}}\right)^{2} \in[6-9] \Rightarrow d_{E . C} & \in[2.45-3] d_{S . C} \\
& \in[3.43-4.2] \mathrm{m} .
\end{aligned}
$$

Además, la recomendación para el grosor del anillo de entrada, la curvatura que debe tener la admisión, indica que debe tener entre $[1 / 5-3 / 8]$ del diámetro de la cámara de estabilización; se asume un grosor de anillo de entrada de $0.75 \mathrm{~m}$, por lo que la sección de entrada es de $d_{E . C}=4.2+0.75=4.950 \mathrm{~m}$.

La longitud de la zona de contracción se elige en base a consideraciones constructivas y recomendaciones [4] que indican que el rango de la longitud de contracción fluctúa entre 0.9 y 1.8 veces el diámetro de entrada al colector, las longitudes más cortas causan irregularidades en el flujo a la salida. Así, $l_{C} \in[0.9-1.8] d_{E, C}=[0.9-1.8] \times 4.95=$ [4.95 - 8.91] m optándose por una longitud de $4.5 \mathrm{~m}$. 
La zona de estabilización está conformada por una sola sección, donde es instalada una rejilla de alambres de forma circular, para atenuar las irregularidades y las turbulencias generadas por la entrada del flujo de aire al colector. Esta malla de alambre, está instalada inmediatamente después de la sección de entrada al colector. El colector se muestra en la Fig. 3. donde la solución propuesta asume que en un primer tramo, con longitud de $2 \mathrm{~m}$, el colector tenga una sección de forma cuadrada, luego en una longitud de dos metros se efectuará la transición suave de la forma cuadrada a la circular, evitando incrementar las pérdidas hidráulicas y finalmente, se tiene una sección circular de longitud de $50 \mathrm{~cm}$ en la salida. De esta forma, el flujo saliente está reconformado con una sección similar a la del modelo.

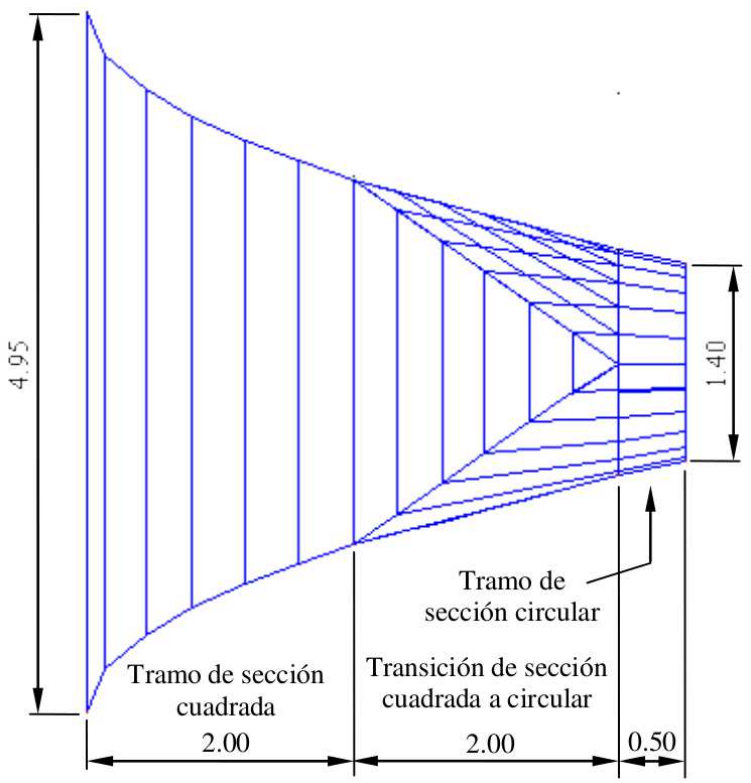

Figura 3: Detalle de la geometría del colector del túnel de viento.

Esta solución tiene la ventaja que ayuda a disminuir las pérdidas en las esquinas de la sección cuadrada, además de uniformizar el flujo para que al incidir sobre el modelo, pueda bañarlo en igual proporción respecto al eje de giro, el modelo alineado con el eje del túnel de viento.

\section{Consideraciones para el diseño del difusor}

El difusor es una parte importante del túnel de viento, pues aquí el flujo comienza a perder velocidad reponiendo la presión. La reducción de la velocidad es necesaria ya que el flujo es expulsado al medio ambiente, grandes velocidades pueden provocar cargas considerables en los apoyos del sistema, debido al efecto de acción y reacción, y provocar altos niveles de ruido y vibración con la consiguiente contaminación sonora. Se optó por diseñar un difusor con sección de entrada un poco mayor que la sección de salida del colector, tomando como diámetro de entrada al difusor $d_{D}=1.45 \mathrm{~m}$.

Según la información bibliográfica [4 6], los difusores son muy sensibles a los errores de diseño, puede causar vibración en el túnel, oscilación del ventilador y variación en la velocidad de la sección de pruebas. La geometría de los difusores en túneles de viento es de forma de cono truncado, en donde el área de cada siguiente sección es mayor que la anterior. Generalmente se utiliza el concepto de ángulo de abertura del difusor. Según algunos investigadores [7, 12] este ángulo no debe exceder de $7^{\circ}$. Otros recomiendan que para difusores de túneles de viento el ángulo de expansión del difusor no debe exceder de $5^{\circ}$ [5]. Para el presente trabajo, se decidió tomar un ángulo de expansión del difusor de $5^{\circ}$ en una longitud de 10 metros, limitado por las condiciones de espacio del lugar de instalación. El difusor tendrá sección circular en toda su longitud y en alguna sección de ella, se instalará el equipo de fuerza, el ventilador, encargado de impulsar el aire a través del túnel de viento.

\section{Cálculo aerodinámico de los componentes del tunel}

En primer lugar, es necesario determinar preliminarmente el gasto másico a través del túnel, bajo las condiciones del diseño. Tomando los datos en la sección de salida del colector, donde $V=14 \mathrm{~m} / \mathrm{s}, d_{S . C}=1.4 \mathrm{~m}$ y la densidad del aire ambiental $\rho=1.225 \mathrm{~kg} / \mathrm{m}^{3}$, utilizando la expresión de continuidad en esta sección afectado por un coeficiente de descarga $C_{D}=0.983$, se tiene:

$$
\bar{m}=C_{D} \rho A V=25.96 \mathrm{~kg} / \mathrm{s} .
$$

Asumiendo que este gasto másico se hace crítico para alguna sección del túnel de viento, se puede escribir

$$
\begin{gathered}
\dot{m}_{m x}=\dot{m}^{*}=\frac{A^{*} p_{o}}{\sqrt{R T_{o}}} \sqrt{k\left(\frac{2}{k+1}\right)^{\frac{k+1}{k-1}}} \Rightarrow \\
A^{*}=\frac{\dot{m}^{*} \sqrt{R T_{o}}}{p_{o}} \sqrt{\frac{1}{k}\left(\frac{k+1}{2}\right)^{\frac{k+1}{k-1}}} .
\end{gathered}
$$

Sustituyendo los datos conocidos se obtiene $A^{*}=0.1085$ $\mathrm{m}^{2}$.

Los parámetros críticos del flujo son

$$
\begin{aligned}
T^{*} & =T_{0}\left(\frac{2}{k+1}\right)=244.2 \mathrm{~K} \\
p^{*} & =p_{0}\left(\frac{2}{k+1}\right)^{\frac{k}{k+1}}=53.528 \mathrm{kPa} \\
\rho^{*} & =\rho_{0}\left(\frac{2}{k+1}\right)^{\frac{1}{k+1}}=0.776 \mathrm{~kg} / \mathrm{m}^{3} .
\end{aligned}
$$


La velocidad crítica del flujo, para las condiciones dadas, es

$$
a^{*}=\sqrt{k R T^{*}}=313.24 \mathrm{~m} / \mathrm{s} .
$$

La secuencia de cálculo para el diseño de las características del tunel de viento la describimos a continuación. i) Cada elemento a calcular, colector y difusor, se divide en tramos menores, por ejemplo, con $\Delta l=0.50 \mathrm{~m}$ y se determina el área para cada sección i-te. ii) Se calcula el coeficiente de gasto $q$ para cada sección i-te, con la relación $q_{i} A^{*} / A_{i}$. iii) Luego, se determina el coeficiente de velocidad $\lambda_{i}$ para cada sección i-te, con ayuda de la siguiente relación

$$
q_{i}=\left(\frac{k+1}{2}\right)^{\frac{1}{k-1}}\left(1-\frac{k+1}{k+1} \lambda_{i}^{2}\right)^{\frac{1}{k-1}} \lambda_{i} .
$$

Esta expresión tiene dos raíces para $\lambda_{i}$, debiéndose tomar el valor del régimen subsónico, $\lambda_{i}<1.0$. El valor supersónico es desechado, puesto que en el túnel las velocidades alcanzadas son subsónicas. iv) A continuación, se determina la velocidad del flujo en la sección i-te con $V_{i}=\lambda_{i} a^{*}$. v) También se calculan los parámetros de presión, temperatura y densidad, para cada sección i-te, con ayuda de las siguientes relaciones,

$$
\begin{aligned}
T_{i} & =T_{o}\left(1-\frac{k-1}{k+1} \lambda_{i}^{2}\right) \\
p_{i} & =p_{o}\left(1-\frac{k-1}{k+1} \lambda_{i}^{2}\right)^{\frac{k}{k-1}}, \\
\rho_{i} & =\rho_{o}\left(1-\frac{k-1}{k+1} \lambda_{i}^{2}\right)^{\frac{1}{k-1}} .
\end{aligned}
$$

En este caso, los parámetros hallados en este punto, corresponde a un proceso adiabático e isoentrópico. Por lo tanto, estos parámetros son teóricos, específicamente en lo que se refiere al cálculo de la caída de presión a lo largo de cada componente del túnel. vi) Se calcula la presión dinámica para cada sección i-te, siendo $p_{\operatorname{din}-i}=\rho_{i} V_{i}^{2} / 2$. vii) Se procede a calcular el número de Reynolds para la sección en análisis $R e=D_{H} V / v$. viii) Con el número $R e$, se calcula el factor de fricción, utilizando la ecuación apropiada para el régimen del flujo. Se recomienda utilizar la ecuación de Colebrook, que no introduce errores sensibles.

$$
\frac{1}{\sqrt{f}}=-2 \log \left(\frac{\varepsilon}{3.7 D_{H}} \frac{2.51}{R e \sqrt{f}}\right) .
$$

ix) Para dos secciones contiguas del elemento dado, se determina el coeficiente de pérdida de presión $K_{\text {perd, ya sea }}$ para contracciones o expansiones continuas, mediante la expresión $K_{\text {perd }}=f L / D$. x) A continuación, se determina la caída de presión para cada tramo determinado en el punto anterior,

$$
\Delta p_{i}=K_{\text {perd }} \frac{\rho_{i} V_{i}^{2}}{2}
$$

Siendo los valores $\rho_{i}$ y $V_{i}$ los correspondientes a la sección de salida de cada tramo. xi) La caída de presión en todo el elemento, es la suma de las caídas parciales de presión $\Delta p=\sum \Delta p_{i}$. xii) La caída de presión $\Delta p$ se transforma a la presión de una columna de agua usando $1 \mathrm{~Pa} \approx 0.102$ $\mathrm{mmH}_{2} \mathrm{O}$. xiii) $\mathrm{Si}$ el elemento analizado no tiene una sección circular, adicionalmente se deberá calcular la pérdida de presión por las esquinas, calculando primero el coeficiente de pérdida por medio de

$$
K_{\text {esq }}=0.10+\frac{4.55}{\left(\log _{10} R e\right)^{2.58}},
$$

luego, la caída total de presión en el elemento no circular será la suma de todas las caídas parciales de presión.

\section{Caída de presión en el colector}

En la Tabla 1 se muestran los resultados del cálculo de las pérdidas por fricción para el colector del túnel de viento, siguiendo la secuencia de cálculo explicada en la subsección anterior. El colector fue dividido en 9 partes, generando 10 secciones. Para el tramo de sección cuadrada y para el tramo de transición se calcularon las pérdidas por fricción y las pérdidas por los efectos de esquina. Como se puede observar, el mayor componente de pérdida se debe al efecto de esquina, el cual es mayor que la pérdida debido a la fricción.

En el colector se instala una malla para estabilizar el flujo. Esta malla también causa una caída de presión, la cual es calculada con la expresión [5]:

$$
\begin{aligned}
k_{g} & =k_{0}+\frac{55.2}{R e_{D}} ; \\
k_{0} & =\left(\frac{1-0.95 \beta}{0.95 \beta}\right)^{2} ; \\
\beta & =\frac{\text { área abierta }}{\text { área total }}=\left(1-\frac{D}{M}\right)^{2} .
\end{aligned}
$$

La pantalla está conformada por una malla de alambre de sección circular de $D=3.175 \mathrm{~mm}$ que genera una maIla cuadrada con lado $M=25.4 \mathrm{~mm}$. Entonces, el coeficiente de área $\beta=0.7656$ y $k_{0}=0.1405$. Así, $k_{g} \approx 0.141$ y la caída de presión debido a la malla estabilizadora es

$$
\Delta p_{g}=K_{g} \frac{\rho_{1} V_{1}^{2}}{2}=0.2 \mathrm{~Pa}=0.021 \mathrm{mmH}_{2} \mathrm{O} \text {. }
$$

\section{Caída de presión en la zona de prueba}

La resistencia que encuentra el flujo al ingresar a la zona de prueba se asemeja a una expansión brusca y el coeficiente de pérdida [12] puede ser hallado en función de la razón del diámetro menor $D_{H 1}$ respecto al diámetro mayor $D_{H 2}$, mediante la expresión

$$
K_{\mathrm{EXP}}=\left[1-\left(\frac{D_{H 1}}{D_{H 2}}\right)^{2}\right]^{2}=0.7046 .
$$


Además, existe una resistencia suplementaria por que el ducto del colector no termina directamente en la sección de entrada a la zona de prueba si no que está embutido en ella, en una distancia de $L=1.0 \mathrm{~m}$. Igual situación se observa en el lado de la entrada del difusor. Esto provoca una caída de presión adicional que es función de la longitud embutida del tubo respecto a su diámetro y del espesor de la pared del tubo respecto a su diámetro [13]. En la Fig. 4 se muestra el esquema de un conducto embutido en una cámara plena.

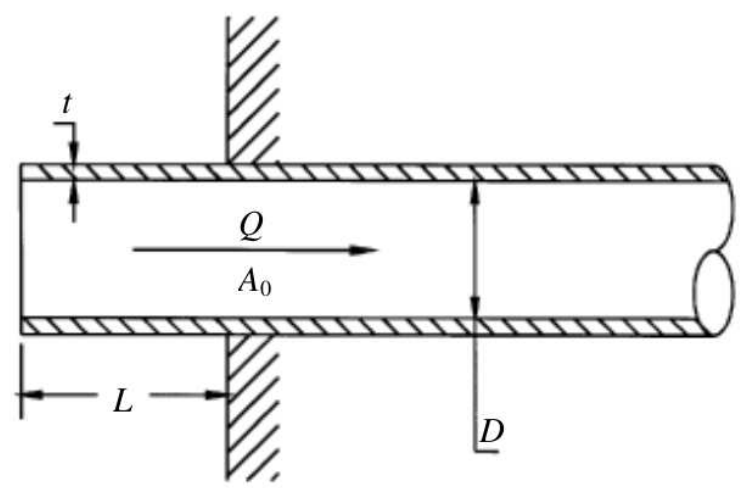

Figura 4: Esquema para la pérdida para ductos embutidos en cámaras plenas.

El coeficiente de pérdida es una función de $t / D$ y $L / D$, y están tabulados para diferentes condiciones. Para el presente caso $t / D_{H 1}=0.001$ y $L / D=0.714$, lo cual da un coeficiente $K_{\text {duc }} \approx 0.9$ [13]. Este coeficiente se multiplica por dos, considerando la entrada del colector y la salida del difusor.

De igual manera, una vez que el fluido pasa por la zona de prueba, para ingresar al ducto del difusor ocurre una pérdida, que es similar a una contracción brusca. Para este caso, el coeficiente de pérdida esta dado por [12],

$$
K_{\mathrm{CONT}}=0.5\left[1-\left(D_{H 1} / D_{H_{2}}\right)^{2}\right]=0.42 .
$$

Así, la caída de presión total observada en la zona de prueba está dada por

$$
\begin{aligned}
\Delta p_{Z P} & =\left(2 K_{d u c}+K_{Z P}\right) \frac{\rho V^{2}}{2} \\
& =363 \mathrm{~Pa}=37 \mathrm{mmH}_{2} \mathrm{O} .
\end{aligned}
$$

Durante la operación del túnel de viento, el modelo se encuentra instalado en la zona de prueba, por lo tanto, su presencia causa una caída de presión. No existen recomendaciones o datos experimentales que evalúen estas pérdidas por lo que éstas deben asumirse en función de la complejidad de la resistencia que el modelo ofrezca al paso del flujo. Para el presente caso, se asumió que el aerogenerador ocasionará una resistencia que puede ser proporcional al coeficiente $K_{M}=0.5$, considerando que las palas del rotor fueron diseñadas aerodinámicamente para ofrecer menor resistencia al paso del viento, por lo que

$$
\begin{aligned}
\Delta p_{M} & =K_{M} \frac{\rho V^{2}}{2} \\
& =62.04 \mathrm{~Pa}=6.32 \mathrm{mmH}_{2} \mathrm{O} .
\end{aligned}
$$

\section{Caída de presión en el difusor}

La longitud total del difusor es $L_{D}=10 \mathrm{~m}$ y el ángulo de abertura adoptado es de $\alpha=5^{\circ}$. De acuerdo a la Ec. (14) se tiene que

$$
\begin{aligned}
\frac{p_{01}}{p_{02}} & =\frac{A_{2}}{A_{1}} \frac{q_{2}}{q_{1}} \Rightarrow \frac{q_{E D}}{q_{S C}}=\frac{A_{S C}}{A_{E D}}=\left(\frac{d_{S C}}{d_{E C}}\right)^{2} \\
q_{E D} & =0.06678,
\end{aligned}
$$

la nueva sección crítica para el flujo en el difusor es

$$
q_{E D}=A_{D}^{*} / A_{E D} \Rightarrow A_{D}^{*}=0.1103 .
$$

y la nueva sección crítica para el flujo en el difusor es

$$
q_{E D}=A_{D}^{*} / A: E D \Rightarrow A_{D}^{*}=0.1103
$$

Para efectuar el cálculo aerodinámico del difusor, este fue dividido en 10 tramos de longitud $L=10$ m cada uno, sin contar el tramo donde estará instalado el ventilador. Así, cada área posterior es calculada mediante

$$
D_{i+1}=D_{i}+2 L \tan (\alpha / 2)
$$

y el coeficiente de pérdida en el difusor se calcula mediante

$$
K_{D}=\left[\frac{f}{8 \tan (\alpha / 2)}+0.6 \tan (\alpha / 2)\right]
$$

De manera similar al cálculo del colector se realiza el cálculo de la caída de presión debido a la fricción que se tiene en el difusor. Los resultados se presentan en la Tabla 2

En realidad, el difusor puede ser dividido en dos partes. Una primera parte, hasta la sección de entrada del ventilador, que trabaja a régimen de succión, con presión negativa, y otro segundo tramo, desde la sección de salida del ventilador hasta la sección de salida del difusor, que trabaja a sobrepresión, presión estática positiva.

La caída de presión en todo el sistema del túnel de viento es igual a la suma de caídas de presión en cada uno de sus elementos,

$$
\begin{aligned}
\Delta p_{\text {Total }} & =\Delta p_{c}+\Delta p_{Z P}+\Delta p_{M}+\Delta p_{D} \\
& =481.2 \mathrm{~Pa}=49.06 \mathrm{~mm} \mathrm{H}_{2} \mathrm{O} .
\end{aligned}
$$




\section{Dimensionamiento del ventilador}

La potencia requerida por el ventilador se calcula mediante [13],

$$
P=\frac{Q p_{s}}{1000 \eta}=\frac{\dot{m} p_{s}}{1000 \rho \eta}=14.4 \mathrm{~kW} .
$$

La alternativa de fabricar un ventilador a la medida era demasiado costoso comparado con la alternativa de elegir uno de los ventiladores ya desarrollados por ITSA. Es evidente, que la elección lleva consigo el riesgo de sobredimensionar el ventilador, lo cual puede traer consecuencias económicas durante la operación del túnel de viento. Como era la solución más viable, se optó por elegir un ventilador axial ITSA con las siguientes características: a) caudal a libre descarga, 69,000 CFM; b) caudal de 2 pulgadas de columna de agua, 50,000 CFM; c) Motor eléctrico trifásico de $30 \mathrm{HP}$, d) diámetro externo del ventilador axial, $1.50 \mathrm{~m}$; e) Medida longitudinal del ventilador, $930 \mathrm{~mm}, \mathrm{f}$ ) número de álabes del ventilador, 17.

Una vez determinadas las medidas geométricas de los elementos que componen el túnel se procedió a elaborar el plano de la vista general de la instalación, el cual se muestra en la Fig. 5 que muestran también las medidas referenciales del citado laboratorio.

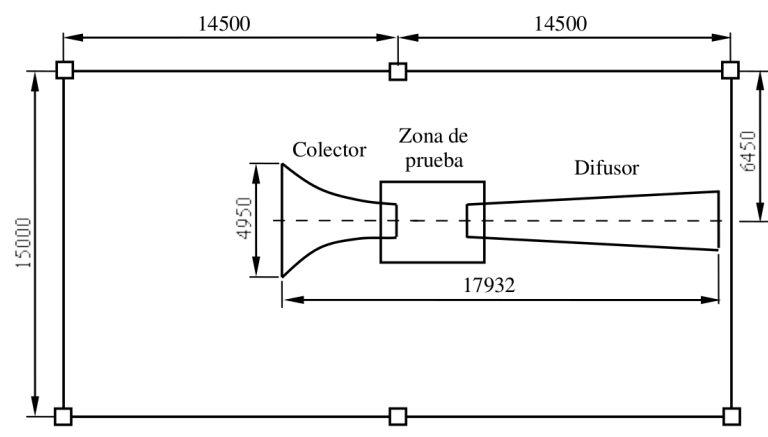

Figura 5: Vista superior de la instalación del túnel de viento.

\section{Conclusiones}

El túnel de viento proyectado es de tipo Eiffel, de circuito abierto y zona de prueba abierta, para ensayar modelos de un diámetro de un metro y velocidades de viento máxima de $14 \mathrm{~m} / \mathrm{s}$.

El túnel consta de 4 partes importantes, el colector con la rejilla de estabilización, la zona de prueba y el difusor. El equipamiento principal está conformado por un ventilador y un variador electrónico de velocidad.

Dada la baja velocidad del flujo de aire en las secciones a través del túnel de viento, el efecto de compresibilidad es mínimo, por lo que bien hubiera sido posible diseñar aerodinámicamente el túnel considerando al flujo de aire como incompresible. Las variaciones de densidad son mínimas como muestran los cálculos efectuados en este trabajo.

Para determinar la forma del colector, se asumió una contracción continua, sin cambio en la curva del perfil del colector, siendo esta de forma cóncava lo que redujo muchos efectos contrarios, como la compresibilidad, desprendimiento dentro del túnel y formación de inestabilidades y fuerte turbulencia.

Dada la baja velocidad de entrada al colector, no fue necesario instalar una zona de estabilización (honeycombs) ya que sólo se requirió de una rejilla o malla de alambre como zona de estabilización.

Las caídas de presión más significativas se encuentran en la zona de prueba, debido a varios fenómenos asociados a su configuración (zona de prueba abierta con similaridad a expansión brusca a la salida del colector y contracción brusca a la entrada del difusor, además de la presencia del modelo). En esta zona se calculó una caída de cerca de 40 $\mathrm{mm}$ de $\mathrm{H}_{2} \mathrm{O}$, contra los 10 restantes en otros elementos del túnel de viento. La pérdida total de presión fue cerca de $50 \mathrm{~mm} \mathrm{H}_{2} \mathrm{O}$.

Al estar el ventilador de tipo axial ubicado pasando la zona de prueba, el túnel trabaja en un régimen de succión, con presión negativa corriente arriba del ventilador y presión positiva corriente abajo del ventilador.

El difusor no pudo ser diseñado para llevar la velocidad de salida hasta valores cercanos a cero, debido a las limitaciones de espacio que restringían la longitud del difusor. Esto provocó que se tenga un difusor corto, el aire a la salida tiene valores cercanos de $4.5 \mathrm{~m} / \mathrm{s}$ lo cual provoca ruido relativamente alto por la descarga a velocidad relativamente alta.

Se pudo observar durante la etapa de pruebas de funcionamiento, que en la zona de prueba con condición del sistema de aerogeneración instalado, la velocidad máxima alcanzada por el flujo de aire fue de $14.60 \mathrm{~m} / \mathrm{s}$, que es un poco mayor a la velocidad de diseño igual a $14 \mathrm{~m} / \mathrm{s}$. Esto se debe a que como fue remarcado, no existe en el mercado nacional condiciones para la construcción de grandes ventiladores sobre medida y tuvo que elegirse un ventilador muy cercano a las necesidades, pero que tiene una designación de ventilación minera.

Para el caso de la prueba de funcionamiento en vacío, la velocidad que alcanza el flujo de aire en el ventilador es mayor que en la conclusión anterior, superando los 15 $\mathrm{m} / \mathrm{s}$. Esto se debe a que la presencia del ventilador causa una caída de presión (parte de la energía es transformada en energía mecánica).

El túnel fue testado en condiciones de diseño, instalándose y ensayándose un aerogenerador de $50 \mathrm{~W}$, para obtener la curva de potencia; obteniéndose resultados cercanos a los teóricos, expuestos en aerogeneración. 


\begin{tabular}{|c|c|c|c|c|c|c|c|c|c|c|}
\hline Sección & 0 & 1 & 2 & 3 & 4 & 5 & 6 & 7 & 8 & 9 \\
\hline$D_{H}(\mathrm{~m})$ & 4.950 & 3.767 & 3.277 & 2.901 & 2.584 & 2.304 & 2.052 & 1.820 & 1.603 & 1.400 \\
\hline$A\left(\mathrm{~m}^{2}\right)$ & 24.503 & 14.189 & 10.737 & 8.414 & 6.675 & 5.025 & 3.758 & 2.778 & 2.019 & 1.540 \\
\hline$q$ & 0.00450 & 0.00778 & 0.01028 & 0.01312 & 0.01653 & 0.02196 & 0.02936 & 0.03972 & 0.05465 & 0.07164 \\
\hline$\lambda$ & 0.00285 & 0.00493 & 0.00652 & 0.00832 & 0.01048 & 0.01392 & 0.01862 & 0.02519 & 0.03465 & 0.04544 \\
\hline$V(\mathrm{~m} / \mathrm{s})$ & 0.89 & 1.54 & 2.04 & 2.61 & 3.28 & 4.36 & 5.83 & 7.89 & 10.85 & 14.23 \\
\hline$T(\mathrm{~K})$ & 293.0 & 293.0 & 293.0 & 293.0 & 293.0 & 293.0 & 293.0 & 293.0 & 292.9 & 292.9 \\
\hline$p(\mathrm{kPa})$ & 101.325 & 101.324 & 101.322 & 101.321 & 101.319 & 101.314 & 101.305 & 101.287 & 101.254 & 101.203 \\
\hline$\rho\left(\mathrm{kg} / \mathrm{m}^{3}\right)$ & 1.225 & 1.225 & 1.225 & 1.225 & 1.225 & 1.225 & 1.225 & 1.225 & 1.224 & 1.224 \\
\hline$p_{V}(\mathrm{~Pa})$ & 0.488 & 1.460 & 2.554 & 4.159 & 6.599 & 11.642 & 20.830 & 38.119 & 72.109 & 123.967 \\
\hline$R e \times 10^{-5}$ & 3.0265 & 3.9839 & 4.5833 & 5.1774 & 5.8087 & 6.8812 & 8.1959 & 9.8328 & 11.9187 & 13.6512 \\
\hline$f$ & 0.0147 & 0.0141 & 0.01385 & 0.01365 & 0.0135 & 0.01328 & 0.01309 & 0.01295 & 0.01284 & 0.01288 \\
\hline$K_{\text {esq }}$ & 0.156 & 0.153 & 0.152 & 0.151 & 0.150 & 0.148 & 0.146 & 0.145 & 0.143 & 0.142 \\
\hline$\Delta p_{f}(\mathrm{~Pa})$ & 0.00072 & 0.00273 & 0.0054 & 0.00979 & 0.01724 & 0.03355 & 0.06645 & 0.13565 & 0.28873 & 0.5701 \\
\hline$\Delta p_{\text {esq }}(\mathrm{Pa})$ & 0.0764 & 0.2241 & 0.3881 & 0.6269 & 0.9872 & 1.7229 & 3.0498 & 5.5215 & 10.3315 & 17.6294 \\
\hline$\Delta p_{\text {parc }}(\mathrm{Pa})$ & 0.0771 & 0.2268 & 0.3935 & 0.6367 & 1.0044 & 1.7565 & 3.1163 & 5.6572 & 10.6202 & . \\
\hline$\Delta p_{\text {total }}(\mathrm{Pa})$ & 23.49 & . & . & . & . & . & . & . & . & . \\
\hline$\Delta p_{\text {total }}\left(\mathrm{mm} \mathrm{H}_{2} \mathrm{O}\right)$ & . & . & . & 2.39 & . & . & . & . & . & . \\
\hline
\end{tabular}

Tabla 1: Resultados del cálculo aerodinámico del colector.

\begin{tabular}{|c|c|c|c|c|c|c|c|c|c|c|c|}
\hline Sección & 0 & 1 & 2 & 3 & 4 & 5 & 6 & 7 & 8 & 9 & 10 \\
\hline$D_{H}(\mathrm{~m})$ & 1.450 & 1.537 & 1.625 & 1.712 & 1.799 & 1.886 & 1.974 & 2.061 & 2.148 & 2.235 & 2.323 \\
\hline$A\left(\mathrm{~m}^{2}\right)$ & 1.651 & 1.856 & 2.073 & 2.301 & 2.542 & 2.795 & 3.059 & 3.336 & 3.624 & 3.925 & 4.237 \\
\hline$q$ & 0.06679 & 0.05942 & 0.05321 & 0.04792 & 0.04339 & 0.03946 & 0.03605 & 0.03306 & 0.03043 & 0.02810 & 0.02603 \\
\hline$\lambda$ & 0.04237 & 0.03769 & 0.03374 & 0.03038 & 0.02751 & 0.02502 & 0.02286 & 0.02096 & 0.01929 & 0.01781 & 0.0165 \\
\hline$V(\mathrm{~m} / \mathrm{s})$ & 13.27 & 11.81 & 10.57 & 9.52 & 8.62 & 7.84 & 7.16 & 6.57 & 6.04 & 5.58 & 5.17 \\
\hline$T(\mathrm{~K})$ & 292.9 & 292.9 & 292.9 & 293.0 & 293.0 & 293.0 & 293.0 & 293.0 & 293.0 & 293.0 & 293.0 \\
\hline$p(\mathrm{kPa})$ & 101.219 & 101.241 & 101.258 & 101.270 & 101.280 & 101.288 & 101.294 & 101.299 & 101.303 & 101.306 & 101.309 \\
\hline$\rho\left(\mathrm{kg} / \mathrm{m}^{3}\right)$ & 1.224 & 1.224 & 1.224 & 1.225 & 1.225 & 1.225 & 1.225 & 1.225 & 1.225 & 1.225 & 1.225 \\
\hline$p_{V}(\mathrm{~Pa})$ & 107.794 & 85.310 & 68.373 & 55.438 & 45.462 & 37.607 & 31.395 & 26.394 & 22.356 & 19.058 & 16.358 \\
\hline $\operatorname{Re} \times 10^{-5}$ & 13.1802 & 12.4300 & 11.7590 & 11.1567 & 10.6178 & 10.1251 & 9.6790 & 9.2669 & 8.8897 & 8.5411 & 8.2218 \\
\hline$f$ & 0.01287 & 0.01285 & 0.01284 & 0.01284 & 0.01285 & 0.01286 & 0.01288 & 0.01290 & 0.01292 & 0.01295 & 0.01297 \\
\hline$K_{D}$ & 0.06305 & 0.06299 & 0.06296 & 0.06296 & 0.06299 & 0.06302 & 0.06308 & 0.06314 & 0.06319 & 0.06328 & 0.06334 \\
\hline$\Delta p_{D}(\mathrm{~Pa})$ & 6.80 & 5.37 & 4.31 & 3.49 & 2.86 & 2.37 & 1.98 & 1.67 & 1.41 & 1.21 & 1.04 \\
\hline$\Delta p_{\text {total }}(\mathrm{Pa})$ & 32.50 & & & & & & & & & & \\
\hline$\Delta p_{\text {total }}\left(\mathrm{mm} \mathrm{H}_{\mathbf{2}} \mathrm{O}\right)$ & 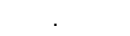 & & . & 3.31 & . & . & & . & & . & \\
\hline
\end{tabular}

Tabla 2: Resultados del cálculo aerodinámico del difusor. 


\section{Referencias}

[1] D. D. Baals y W. R. Corliss; Wind Tunnels of NASA, Scientific and Technical Information Branch, NASA SP 440, USA (1981). Disponible en: http://www.hq.nasa.gov/office/pao/History /SP-440/contents.htm accesado el 08 abril 2014.

[2] J. Katz, Annu. Rev. Fluid. Mech. 38, 27 (2006).

[3] J. B. Barlow, W. H. Rae y A. Pope; Low speed wind tunnel testing, Wiley \& Sons, New York (1999).

[4] J. H. Bell y R. D. Mehta; Contraction design for small low-speed wind tunnel, NASA CR-177488, Washington D.C., National Aeronautics and Space Administration (1988).

[5] R. D. Mehta, P. Bradshaw; The Aeronautical Journal of the Royal Aeronautical Society, Technical Notes, pp. 443-449, November (1999).

[6] A. L. Pope y K. Goin; Wind-tunnel Testing, Willey \& Sons, New York (1965).
[7] I. Prada y Nogueira, El túnel de viento como herramienta de ensayo aerodinámico en la Fórmula I, Anales de Mecánica y Electricidad, Asociación de Ingenieros del ICAI, Madrid, Nov-Dic (2006).

[8] M. J. Zucrow y J. D. Hoffman; Gas Dymanic, Willey \& Sons, New York (1976).

[9] G. C. Oates, Aerothermodynamics of Gas Turbine and Rocket propulsión, American Institute of Aeronautics and Astronautics, USA (1984).

[10] M. E. Deich y A. E. Zariankin; Hidrogasodinámica, Ed. Energoatomizdat, Moscú (1984).

[11] P. M. Gerhart, R. J. Gross y J. I. Hochstein; Fundamentos de Mecánica de Fluidos, Addison-Wesley lberoamericana, México (1995).

[12] Crane Co, Engineering Department; Flow of Fluids through valves, fittings and pipe, USA (1988).

[13] ASHRAE Handbook - Fundamentals (SI), American Society of Heating Refrigerating and Air-Conditioning Engineers, USA (2005). 